\title{
Antimicrobial Use in the ICU: Indications and Accuracy-An Observational Trial
}

\author{
Phillip D. Levin, MB, BChir ${ }^{1 \star}$, Suhel Idrees, MD, Charles L. Sprung, MD¹, Charles Weissman, MD, Yoram Weiss, MD, \\ Allon E. Moses, MD², Shmuel Benenson, MD²
}

${ }^{\top}$ Department of Anesthesiology and Critical Care Medicine, Hadassah Hebrew University Medical Center, Jerusalem, Israel; '2Department of Clinical Microbiology and Infectious Diseases, Hadassah Hebrew University Medical Center, Jerusalem, Israel.

BACKGROUND: In intensive care unit (ICU) patients, signs of infection and inflammation are similar, making diagnosis of bacterial infections difficult. Antimicrobials may therefore be overused, contributing to development of antimicrobialresistant bacteria.

OBJECTIVES: To measure the accuracy of clinician decisions to start antimicrobials; to correlate clinician certainty with the presence of infection; and to examine whether physiological variables correlate with clinician certainty.

DESIGN: Prospective observational study.

SETTING AND PATIENTS: Patients staying $>48$ hours in a general ICU of a tertiary care hospital.

MEASUREMENTS: The ICU clinician's certainty for the presence of infection was recorded when starting antimicrobials. An independent infectious diseases (ID) specialist determined if antimicrobials were required and if infection was present. Clinician antibiotic start decisions were tested for accuracy according to the ID determination for the presence of infection.
RESULTS: Empirical antimicrobial therapy was justified by the presence of infection on 67/125 (54\%) occasions. Clinician certainty for infection correlated well with the presence of defined infection $\left(r^{2}=0.78\right)$, however, infection was defined on 6/19 (31\%) occasions when ICU clinician certainty was low $(\leq 2)$, and antimicrobials were prescribed even when clinician certainty was minimal. Antimicrobial course length was similar whether infection was defined or not (11.5 \pm 9.2 vs $10.7 \pm 9.1$ days; $P=0.65$ ). Physiological variables were not associated with clinician certainty of infection.

CONCLUSIONS: Antimicrobial therapy is probably overused in the ICU, possibly resulting from difficulties in diagnosis and the perceived greater risk of untreated infection when compared to the risks of potentially unnecessary antimicrobial therapy. Efforts to improve antimicrobial-related decision-making should be mandatory. Journal of Hospital Medicine 2012;7:672-678. (C) 2012 Society of Hospital Medicine.
Antimicrobial use provides the selective pressure that cause bacteria to develop antimicrobial resistance. ${ }^{1}$ Currently, clones of bacteria with very limited antimicrobial sensitivity are gradually spreading around the world. $^{2}$ The intensive care unit (ICU) is a focus of resistant bacteria within the hospital ${ }^{3}$ as a result of high illness severity, widespread use of invasive monitoring or therapeutic devices, frequency of bacterial infection (found in approximately $51 \%$ of patients ${ }^{4}$ ), and consequent extensive use of broad-spectrum antimicrobials (in $71 \%$ of patients). ${ }^{4}$

When prescribing antimicrobials, the ICU clinician often faces a dilemma. First, the traditional symptoms and signs of infection (such as characteristic patient history, fever, increased white cell count, etc) are com-

\footnotetext{
*Address for correspondence and reprint requests: Phillip D. Levin, Department of Anesthesia and Critical Care Medicine, Hadassah Hebrew University Medical Center, POB 12000, Jerusalem 91120, Israel; Telephone: 9722 6777111; Fax: 97216779392 ; E-mail: phillipl@hadassah.org.il

Additional Supporting Information may be found in the online version of this article.

Received: January 14, 2012; Revised: May 31, 2012; Accepted: June 19,2012

2012 Society of Hospital Medicine DOI 10.1002/jhm.1964

Published online in Wiley Online Library (Wileyonlinelibrary.com).
}

mon in ICU patients even in the absence of infection, making distinction of infectious and noninfectious causes of patient deterioration difficult. Second, delaying antimicrobial therapy, prescribing inadequate antimicrobials, or allowing bacterial infections to go untreated, increases patient mortality, ${ }^{5-7}$ resulting in guideline recommendations to start broad-spectrum antimicrobials as soon as possible in the presence of suspected severe sepsis. ${ }^{8}$ While third, and in contrast, unnecessary antimicrobial therapy increases the risk of antimicrobial-related complications, such as Clostridium difficile colitis (with a crude mortality of up to $20 \%{ }^{9}$ ), and potentially endangers the greater population of ICU patients by increasing the prevalence of resistant organisms. Choosing between delaying necessary antimicrobial therapy and exposing the patient to unnecessary therapy requires that 2 contrasting risks be balanced-that of untreated infection versus late antimicrobial complications.

The main aim of this study was to assess how often administration of antimicrobials for suspected infection could be justified by the presence of infection. The primary outcome measure was accuracy of antimicrobial administration, defined as the proportion of antimicrobials started for suspected infection where infection was later proven to have been present. Secondary 
outcome measures examined: (1) whether clinician suspicion of infection correlated with the presence of defined infection; (2) the ID specialist's accuracy for empiric antimicrobial administration; (3) whether common clinical parameters were associated with clinician certainty regarding the presence of infection; and (4) use of antimicrobials in the presence or absence of infection. These data are important in order to identify possibilities for improving antimicrobial administration.

\section{METHODS}

\section{Setting}

Data were collected on all ICU patients staying $>48$ hours in the 12-bed general (mainly surgical) ICU of a 775-bed academic tertiary referral center (the Hadassah Hebrew University Medical Center, Jerusalem, Israel) from May to August 2009. The hospital ethics committee approved the study and waived the requirement for informed consent.

Clinical antimicrobial decision-making was at the final discretion of the ICU attending clinician. During office hours, decisions to start antimicrobials with any but first line agents (ampicillin, ampicillin/clavulanic acid, azithromycin, cefazolin, cefuroxime, ciprofloxacin, clindamycin, cloxacillin, gentamicin, and metronidazole) required authorization by the clinical ID specialist on attachment to the ICU (who performed a daily round). Out of office hours, decisions required authorization by an on-call ID specialist (usually by phone). There was no availability of a clinical pharmacist. Microbiological studies were obtained as follows: sputum and urine cultures routinely 3 times per week, while other cultures (including blood, wound, site-specific cultures, etc) according to clinical indications.

\section{Antimicrobial Administration Decisions}

Start and stop dates were recorded for all intravenous antimicrobials administered during the patient's ICU stay. Antimicrobial start decisions were divided into 3 groups: empirical (where antimicrobials were started for a new suspected infection), prophylaxis-driven (antimicrobials given peri-procedurally), and targeted therapy (antimicrobials started or changed based on receipt of culture results, or antimicrobials continued from a previous department). Although multiple antimicrobials were often started together, these were considered as a single antimicrobial start decision, if started for the same reason.

Empirical decisions represent the main focus of this study, and further data were collected for these decisions. For each empiric decision, the attending ICU clinician's name was recorded, as well as a measure of his certainty that a new infection was actually present. Certainty was determined at the time that the antimicrobials were started and was entirely subjective. The clinician was asked to categorize his certainty that an infection was present when starting empiric antimicrobials on a scale from 0 to 5: 0-no infection; 1infection unlikely; 2-infection possible; 3-infection probable; 4-infection very likely; and 5-infection certain. The number of systemic inflammatory response syndrome (SIRS) criteria $^{10}$ and Sequential Organ Failure Assessment (SOFA) ${ }^{11}$ score were calculated at the time each decision was made (using the last available data prior to starting antimicrobials), and for the previous 2 days (using the worst values on each calendar day). Data on demographics, admission history, comorbid conditions, Acute Physiology and Chronic Health Evaluation II (APACHE II) score, and outcome were collected for each patient.

\section{Antimicrobial Start Decisions and Definitions of Infection by the Study ID Specialist}

Approximately 1 week after each empiric antimicrobial decision, the need for antimicrobial therapy and the presence of infection were analyzed and defined by the study ID specialist (S.B.). He was not involved in clinical decision-making and was not acquainted with patient details. Each analysis included 2 steps: Step 1 concerning the overall requirement for antimicrobial therapy, and Step 2 regarding the presence of infection. For Step 1, data from the patient's clinical course up until the time the antimicrobial start decision was made were presented. At that point, the study ID specialist decided whether, if presented the case as a consultant, he would have recommended starting antimicrobials. For Step 2, the patient's clinical course following the antimicrobial decision point as well as laboratory, imaging, and microbiological results from the subsequent days were reviewed. The presence or absence of infection was defined by integrating all of this data, and based on the Centers for Disease Control and Prevention (CDC) surveillance criteria for the diagnosis of nosocomial infections. ${ }^{12}$ The study ID specialist used the same certainty score regarding the presence of infection as the clinicians. A certainty of a "probable infection" (score 3) or higher represented the cutoff to define the presence of infection. The study ID specialist's determination of the presence of infection was considered the "gold standard" for the presence of infection for analyses and is termed "defined infection."

\section{Accuracy of Antimicrobial Start Decisions}

Accuracy was calculated for both the clinicians and the study ID specialist, and expressed as a proportion. The denominator for ICU clinician accuracy was the total number of antibiotic start decisions for suspected infection, and the numerator was the number of decisions where infection was defined by the ID specialist. For the study ID specialist, the denominator was the number of occasions when antibiotic administration was considered justified in the Step 1 analysis, and the numerator was the number of these cases where 


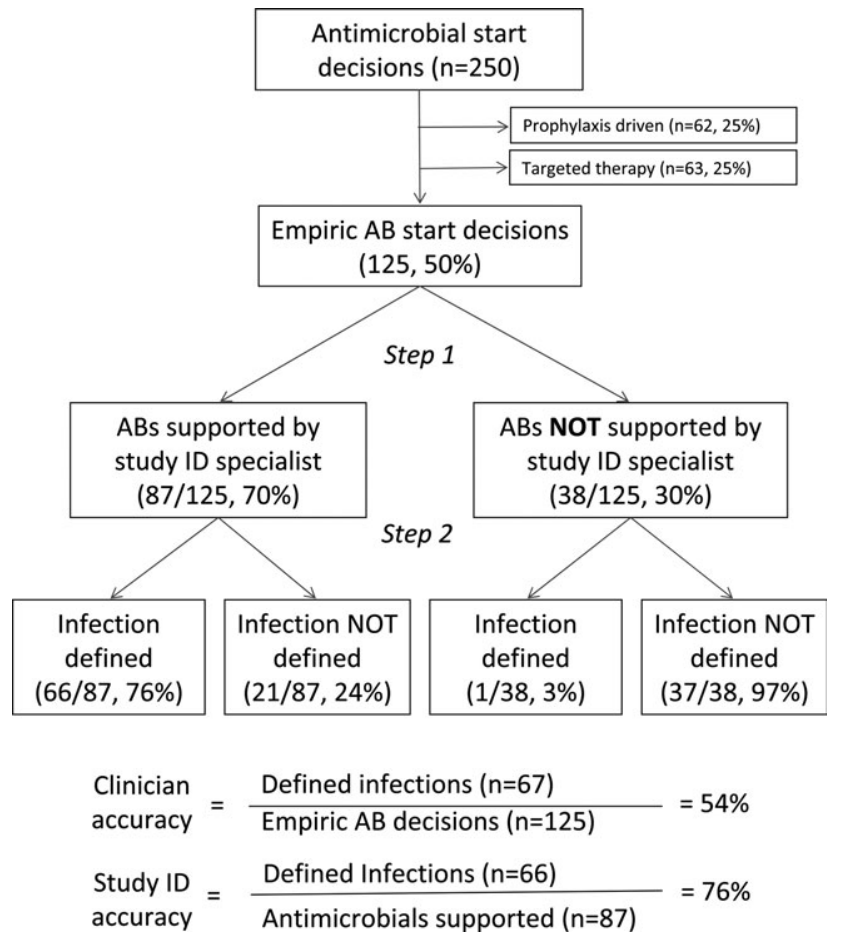

FIG. 1. Flow diagram of study methodology and main results. Abbreviations: $A B$, antimicrobial. ID infectious diseases specialist.

infection was defined (Figure 1). The correlation between clinician certainty and the study-defined presence of infection was examined. The accuracy of clinical antimicrobial decisions made during the first 48 hours of ICU admission was compared to decisions made after 48 hours.

In order to assess the robustness of the study findings, the ICU clinician accuracy was examined in a sensitivity analysis. Accuracy was calculated using a lower cutoff for the study ID specialist's definition of infection- "possible infection" (score 2) or above, rather than "probable infection" or above.

\section{Physiological Parameters}

To examine the effect of physiological variables on physician certainty, empiric antimicrobial start decisions were divided into 2 groups-a high clinician certainty group (certainty score $\geq 3$ ) and a low certainty score $(<3)$. Each physiological parameter comprising the SIRS and SOFA scores, the scores themselves, and changes from the previous 24 and 48 hours were compared for the 2 groups. Data used for the decision day were the last available observations prior to starting the antimicrobials. Data for the previous 2 days were the worst values present during each calendar day.

\section{Antimicrobial Course Length}

The total course given after each empirical antibiotic start decision was measured in days. The course length started with the empiric antimicrobial start decision, and ended either when antimicrobial therapy was stopped, or when a subsequent empirical start de-
TABLE 1. Demographic, Clinical Characteristics, and Outcome of the Study Patients

\begin{tabular}{lc}
\hline & $\begin{array}{c}\text { No. (\%) or Mean } \pm \text { SD } \\
\text { N }=119\end{array}$ \\
\hline Demographics & \\
Male gender & $66(55)$ \\
Age (years) & $53 \pm 25$ \\
Hospital admission prior to ICU admission & $62(52)$ \\
Independent functional capacity & $99(83)$ \\
Etiology for ICU admission" & \\
Surgery & $82(69)$ \\
Elective & $12(10)$ \\
Emergency & $70(59)$ \\
Trauma & $41(34)$ \\
Medical & $26(22)$ \\
Comorbidities & \\
Prior antimicrobial therapy & $48(40)$ \\
Severe cardiac disease ${ }^{\dagger}$ & $12(10)$ \\
Severe respiratory disease ${ }^{\dagger}$ & $5(4)$ \\
Diabetes mellitus & $22(18)$ \\
Liver disease $^{\dagger}$ & $10(8)$ \\
Dialysis & $5(4)$ \\
APACHE $\|$ score & $15 \pm 8$ \\
Outcome & \\
ICU length of stay (days) & $13 \pm 15$ \\
Hospital length of stay (days) & $36 \pm 32$ \\
ICU mortality & $16(13)$ \\
Hospital mortality & $22(18)$ \\
\hline
\end{tabular}

Abbreviations: APACHE, Acute Physiology and Chronic Health Evaluation II; ICU, intensive care unit; SD, standard deviation.

* Patients may have had more than 1 etiology at admission.

${ }^{\dagger}$ As determined by the APACHE $\|$ definitions.

cision was made. Course length for start decisions where infection was subsequently defined was compared to decisions where infection was not defined.

\section{Statistical Analysis}

Continuous variables were compared using the Student $t$ test, while categorical variables were compared using the chi-square test. All $P$ values are 2 -tailed and $P<0.05$ was considered statistically significant. SAS version 8.2 (SAS Institute, Inc, Cary, NC) was used for statistical analysis.

\section{RESULTS}

Data were collected on 119 consecutive ICU patients over 4 months (Table 1). Antimicrobials were started for suspected infection in 80/119 (67\%) patients, for prophylaxis in 55/119 (46\%) patients, and for other reasons in 42/119 (35\%) patients. More than one indication was present during the patient's ICU admission among 41/119 (34\%) patients, while for 6/119 (5\%) patients, no antimicrobials were prescribed at all. Among these patients, antimicrobials were administered on 250 occasions, including 125/250 (50\%) occasions for suspected infection (empirical decisions), 62/250 $(25 \%)$ occasions for procedural prophylaxis (prophylaxis-driven), and on 63/250 (25\%) occasions for other reasons (antimicrobial changes following receipt of culture results, or continuation of antimicrobials 


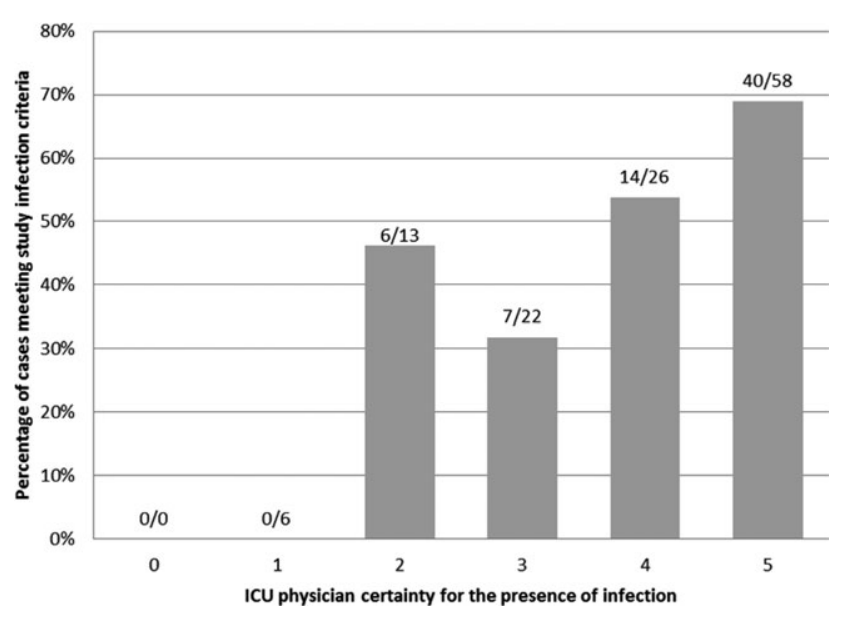

FIG. 2. Correlation between clinician certainty and study-defined presence of infection. Number of defined infection/number of cases in each clinician certainty group are presented above the bars. Abbreviations: ICU, intensive care unit.

prescribed prior to ICU admission). Microbiological cultures were obtained from the study population on 2132 occasions, including 395 blood cultures in which significant organisms (not reflecting contamination) grew on $57 / 395(14 \%)$ occasions.

Among the empiric antimicrobial start decisions, infection was defined by the study ID specialist on $67 /$ $125(54 \%)$ occasions, representing the clinicians' diagnostic accuracy. These infections included 17 (25\%) respiratory, $16(24 \%)$ abdominal, $13(19 \%)$ soft tissue, $11(16 \%)$ blood stream, $6(9 \%)$ urinary, and 4 $(6 \%)$ other infections.

Three attending clinicians treated patients during the study period, and their accuracies were similar (21 infections defined/44 start decisions for suspected infection, $48 \% ; 24 / 38,63 \% ; 22 / 43,51 \%$, for each attending; $P=$ ns for all comparisons). Clinician accuracy was higher for empirical antimicrobial start decisions, made within 48 hours of ICU admission, compared to later decisions (35 defined infections/53 early antibiotic start decisions $[66 \%$ ] vs 32 defined infections/72 late antibiotic start decisions [44\%]; $P=0.02$ ).

In a sensitivity analysis, decreasing the cutoff for the study ID specialist's definition of infection from "probable" (and above) to "possible" (and above) lead to reclassification of $14 / 125(11 \%)$ antimicrobial start decisions from "no infection defined" to "infection defined." This increased physician accuracy from $67 / 125(54 \%)$ to $78 / 125(62 \%)$, and conversely decreased potential antimicrobial overuse from 58/125 $(46 \%)$ to $47 / 125(38 \%)$ decisions $(P=\mathrm{ns})$.

When starting antimicrobials for suspected infection, the clinicians were asked to record their certainty in the presence of infection. Infections were defined on 6/19 $(31 \%)$ occasions when the clinician certainty score was low $(\leq 2)$ versus $61 / 106(57 \%)$ when the clinician certainty score was high $(\geq 3, P=0.037$; Figure 2$)$. Correlation between the clinician certainty score and the presence of defined infection was good $\left(r^{2}=0.78\right)$.
The study ID specialist agreed with the clinician's decision to start antimicrobial therapy on 87/125 $(70 \%)$ occasions. Infection was subsequently defined on $66 / 87(76 \%)$ occasions, representing the study ID specialist's diagnostic accuracy. The study ID specialist's accuracy was significantly higher than the clinician's $(66 / 87$ [76\%] versus 67/125 [54\%]; $P=$ $0.001)$. Notably, there was only 1 case $(3 \%)$ where empiric therapy was deemed unnecessary by the study ID specialist, and where infection was subsequently defined. In this case, the clinicians started antibiotic therapy for suspected ventilator-associated pneumonia in a 66-year-old patient on the 28th day of an ICU admission for head and spinal cord trauma. The ID specialist's certainty for the presence of infection was 3-probable. The patient ultimately survived and was discharged to a rehabilitation facility.

Comparing physiological data for antimicrobial start decisions with high clinician certainty (score $\geq 3$ ) versus low certainty of infection (score $\leq 2$ ), revealed that none of the physiological data, nor changes over time were significantly associated with clinician certainty. Further use of high doses of vasopressors $(>0.1 \mathrm{mcg} /$ $\mathrm{kg} / \mathrm{min}$, SOFA score 4) was present at $42 / 106(40 \%)$ high certainty decisions versus $7 / 19$ (37\%) low certainty decisions $(P=0.819)$. This underscores the physicians' difficulty in distinguishing between infectious and inflammatory causes of deterioration (Table 2).

During the study period, 2541 days of antimicrobial therapy were given of which 1677 (66\%), 413 (16\%), and $451(18 \%)$ were given, respectively, empirically (for suspected infection), for procedural prophylaxis, and as targeted therapy. Antimicrobial course length was $11.5 \pm 9.2$ days in the presence of defined infection versus $10.7 \pm 9.1$ days in the absence of defined infection $(P=0.655)$. Overall, 658/2541 (26\%) days of therapy could potentially have been saved by reducing antimicrobial prescriptions for suspected infections which were not defined.

\section{DISCUSSION}

The use of empirical antimicrobials could be justified by the presence of defined infection on only $54 \%$ of occasions when they were administered, suggesting considerable potential overuse of these drugs. ICU-clinician certainty for the presence of infection correlated well with the number of infections actually defined, however, infections were defined when certainty was low (Figure 2) and antimicrobials prescribed even when clinician certainty was minimal. Common clinical physiological and laboratory parameters did not seem to assist in the clinicians' decision-making, as there were no significant differences in any of these values between empiric decisions with high or low certainty. The study ID specialist showed significantly better accuracy in antimicrobial decision-making than the ICU clinicians. $\mathrm{He}$ agreed with antimicrobial administration on only 
TABLE 2. SIRS and SOFA Score Data Recorded at the Time of Antimicrobial Start for Suspected Infection Plus Changes From the Previous 24 and 48 Hours

\begin{tabular}{|c|c|c|c|}
\hline & $\begin{array}{c}\text { Low Certainty* } \\
\quad \mathrm{N}=19 \\
\text { Mean } \pm \mathrm{SD}\end{array}$ & $\begin{array}{c}\text { High Certainty }^{\dagger} \\
N=106 \\
\text { Mean } \pm \text { SD }\end{array}$ & $P$ Value \\
\hline \multicolumn{4}{|l|}{ SIRS elements } \\
\hline Temperature $\left({ }^{\circ} \mathrm{C}\right)$ & $37.7 \pm 1.2$ & $37.3 \pm 1.6$ & 0.28 \\
\hline WBC count $\left(\times 10^{9} /\right.$ iter $)$ & $16.7 \pm 8.1$ & $15.9 \pm 10.5$ & 0.72 \\
\hline Pulse (rate/min) & $112 \pm 23$ & $110 \pm 21$ & 0.58 \\
\hline Respiratory rate (rate/min) & $22 \pm 8$ & $22 \pm 8$ & 0.90 \\
\hline $\begin{array}{l}\text { Number of SIRS criteria } \\
\text { (at antimicrobial start) }\end{array}$ & $3.0 \pm 0.9$ & $3.2 \pm 0.9$ & 0.24 \\
\hline $\begin{array}{l}\text { Change in number of SIRS } \\
\text { criteria ( } 24 \mathrm{~h} \text { ) }\end{array}$ & $0.1 \pm 0.9$ & $0.0 \pm 0.9$ & 0.68 \\
\hline $\begin{array}{l}\text { Change in number of SIRS } \\
\text { criteria ( } 48 \mathrm{~h} \text { ) }\end{array}$ & $0.0 \pm 0.7$ & $0.3 \pm 0.9$ & 0.36 \\
\hline \multicolumn{4}{|l|}{ SOFA score elements (points) } \\
\hline Respiratory & $1.6 \pm 1.1$ & $1.9 \pm 1.2$ & 0.45 \\
\hline Neurological & $1.8 \pm 1.7$ & $2.0 \pm 1.6$ & 0.50 \\
\hline Coagulation & $0.6 \pm 1.1$ & $0.6 \pm 1.1$ & 0.85 \\
\hline Hepatic & $0.6 \pm 0.8$ & $0.4 \pm 0.8$ & 0.37 \\
\hline Renal & $0.7 \pm 1.1$ & $0.8 \pm 1.1$ & 0.51 \\
\hline Cardiovascular & $1.5 \pm 1.9$ & $1.8 \pm 1.9$ & 0.47 \\
\hline SOFA score day at antimicrobial start & $6.7 \pm 3.1$ & $7.3 \pm 4.6$ & 0.58 \\
\hline SOFA score change (previous $24 \mathrm{~h}$ ) & $1.5 \pm 3.2$ & $1.0 \pm 2.9$ & 0.56 \\
\hline SOFA score change (previous $48 \mathrm{~h}$ ) & $3.1 \pm 4.4$ & $1.5 \pm 4.3$ & 0.25 \\
\hline
\end{tabular}

Abbreviations: SIRS, severe inflammatory response syndrome; SOFA, Sequential Organ Failure Assessment; WBC, white blood cells.

${ }^{*}$ Clinician certainty score for presence of infection 0-2: no infection to possible infection.

${ }^{\dagger}$ Clinician certainty score for the presence of infection 3-5: probable to certain.

$70 \%$ of occasions that clinicians started empiric therapy, and had a higher diagnostic accuracy at a "cost" of only 1 untreated infection.

Two main possibilities are suggested to explain the potential antimicrobial overuse. First, ICU physicians are loath to leave infections untreated and potentially cause immediate increases in mortality. ${ }^{8}$ This leads to uncertainty avoidance or risk aversive behavior that is demonstrated in our study by the inclusion of antimicrobial administration decisions made even when physicians' certainty regarding the presence of infection was low. Uncertainty avoidance has been shown to be significantly associated with antimicrobial prescribing practices, ${ }^{13}$ however, it discounts the risk of antimicrobial complications associated with unnecessary antimicrobial therapy. Second, the diagnosis of infection, and particularly nosocomial infection, in ICU patients is difficult. Symptoms cannot be elicited in obtunded ventilated ICU patients, the physical exam can be equivocal, bacterial growth in cultures (with the exception of blood cultures) often reflects colonization rather than infection, and the laboratory and imaging findings of inflammation and infection are very similar. Our data demonstrated some of these difficulties. Diagnostic accuracy was higher in infections suspected during the first 48 hours of ICU admission when compared to later, presumably as infection leading to ICU admission is associated with symptoms, signs, and an acute change in the patient's condition, factors that may be absent when a patient develops a nosocomial infection. Further, physiological parameters did not correlate with the certainty that ICU clinicians expressed in their decision, indicating the difficulty in interpreting these data. Finally, infection was defined in $30 \%$ of low certainty decisions, indicating that clinical impression alone is not a reliable tool for determining the presence of infection.

Three sets of interventions could be suggested to improve antimicrobial decision-making-increased use of the ID consult, improved laboratory tests for the diagnosis of infection, and a policy of de-escalation. Use of antimicrobial stewardship (often through involvement of an ID physician) reduces antimicrobial usage and the occurrence of resistant bacteria without adverse patient outcomes. ${ }^{14}$ Indeed, our study ID consult showed more accurate antimicrobial prescribing than the ICU clinicians, although he may have been subject to the potential biases described below. All antimicrobial administration decisions taken during the study were, however, made in consultation with the clinical ID consult. The lower performance of the clinical ID consult (when compared to the study ID consult) may have resulted from difficulties in the real-time interaction with the clinicians or from decisions taken during non-office hours. During non-office hours, the on-call ICU resident presented cases to an on-call ID specialist, neither of whom may have been familiar with all the complex case details and therefore may have preferred to err by commission than by omission.

More accurate laboratory tests, such as procalcitonin or real-time bacterial polymerase chain reaction (PCR), ${ }^{15}$ could be beneficial as they might increase physician confidence in decision-making. Procalcitonin has been used in a wide variety of settings ${ }^{16-18}$ (including the $\mathrm{ICU}^{19-21}$ ) to safely decrease antimicrobial starts and/or antimicrobial course length. Despite this, in a large multicenter study of procalcitonin use in ICU patients, ${ }^{19}$ compliance with the antibiotic start protocol was very low. Antibiotics were administered by the participating physicians in $73 / 93(78 \%)$ cases where the procalcitonin tests indicated that antimicrobials were not required, representing protocol violations. In parallel to our study, this demonstrates the reluctance of physicians to abstain from prescribing antimicrobial therapy for suspected infection even when the likelihood of infection may be low.

A strategy of de-escalation offers the possibility of starting broad-spectrum antimicrobials early and, subsequently, narrowing or stopping therapy according to the clinical course and the microbiological results. ${ }^{22,23}$ This strategy allows clinicians to start antimicrobials even when the suspicion of infection is low, but to stop them rapidly as the clinical picture clarifies. Unfortunately, the mean antimicrobial course length in this study was not influenced by the 
presence of infection, indicating that this strategy was not employed successfully.

The proportion of patients prescribed antimicrobials for suspected infection in our study is similar to that found in others (eg, 34\% of patients in a large French surve $\left.{ }^{24}\right)$. The proportion of potentially unnecessary antimicrobials in other studies is also similar, ranging from $14 \%$ to $50 \% .^{24-28}$ In the ICU, the majority of antimicrobial usage studies are microbiology-based and examine whether bacteria cultured are resistant to the antimicrobials chosen. They have shown that inappropriate antimicrobial therapy occurs on $20 \%-$ $36 \%$ of occasions. ${ }^{6,7,29}$ The current study furthers knowledge on antimicrobials decision-making in the ICU, by examining the actual requirement for antimicrobial therapy based on the presence of infection, ie, whether antimicrobials were needed at all.

The principal limitation of the study concerns the determination of the presence of infection. The study premise was that antimicrobials are overused, and this may have biased the study ID consult to underestimate appropriateness of antimicrobial therapy and to define fewer infections. Further, making theoretical decisions in the research office avoids the medical, ethical, and legal issues related to clinical practice, as there is no risk associated with error. This may have allowed the study ID specialist to be overly conservative in his definitions of infections. A wider team of decision-makers to determine the presence of infection, including both ID and ICU specialists, would have lent more weight to their determinations, however, this was logistically impossible. To limit the potential bias, infections were defined as objectively as possible based on the CDC criteria. ${ }^{12}$ Further, the sensitivity analysis showed that while decreasing the study ID specialist's threshold for the definition of infection from "probable" and above to "possible" and above improved physician accuracy, over a third of antimicrobial start decisions remained unjustified by the presence of defined infection. The study was performed in only 1 center and may not reflect general ICU practice, although, as discussed above, the antibiotic decision-making accuracy is in the same orders of magnitude as those found in other somewhat similar studies. Finally, even if unnecessary antimicrobial use was overestimated, the possibility for significant improvement in antimicrobial administration accuracy remains.

In conclusion, our data suggest that on up to $46 \%$ of occasions, empirical antimicrobials are prescribed in the absence of infection. We suggest that the potential antibiotic overuse results from difficulties in diagnosing ICU-related infections, and from the high perceived risk of untreated infection as compared to the risks of potentially unnecessary antimicrobial therapy, representing a type of risk aversive behavior. As antimicrobial use is the primary factor promoting antibiotic resistance and may be a cause of other patient complications, efforts to improve antimicrobial-related decision-making should be mandatory.

Disclosure: This study was presented in part as a poster at the European Society of Intensive Care conference, October 2010. There are no financial conflicts of interest to declare for any author.

\section{References}

1. Gold HS, Moellering RCJr. Antimicrobial-drug resistance. N Engl J Med. 1996;335:1445-1453.

2. Kumarasamy KK, Toleman MA, Walsh TR, et al. Emergence of a new antibiotic resistance mechanism in India, Pakistan, and the UK: a molecular, biological, and epidemiological study. Lancet Infect Dis. 2010;10:597-602.

3. Fridkin SK, Steward CD, Edwards JR, et al. Surveillance of antimicrobial use and antimicrobial resistance in United States hospitals: project ICARE phase 2. Project Intensive Care Antimicrobial Resistance Epidemiology (ICARE) hospitals. Clin Infect Dis. 1999;29: $245-252$.

4. Vincent JL, Rello J, Marshall J, et al. International study of the prevalence and outcomes of infection in intensive care units. JAMA. 2009; 302:2323-2329.

5. Iregui M, Ward S, Sherman G, Fraser VJ, Kollef MH. Clinical importance of delays in the initiation of appropriate antibiotic treatment for ventilator-associated pneumonia. Chest. 2002;122: 262-268.

6. Kollef MH, Sherman G, Ward S, Fraser VJ. Inadequate antimicrobial treatment of infections: a risk factor for hospital mortality among critically ill patients. Chest. 1999;115:462-474.

7. Kumar A, Ellis P, Arabi Y, et al. Initiation of inappropriate antimicrobial therapy results in a fivefold reduction of survival in human septic shock. Chest. 2009;136:1237-1248.

8. Dellinger RP, Levy MM, Carlet JM, et al. Surviving Sepsis Campaign: international guidelines for management of severe sepsis and septic shock: 2008. Crit Care Med. 2008;36:296-327.

9. Gasperino J, Garala M, Cohen HW, Kvetan V, Currie B. Investigation of critical care unit utilization and mortality in patients infected with Clostridium difficile. J Crit Care. 2010;25:282-286.

10. American College of Chest Physicians/Society of Critical Care Medicine Consensus Conference: definitions for sepsis and organ failure and guidelines for the use of innovative therapies in sepsis. Crit Care Med. 1992;20:864-874.

11. Vincent JL, Moreno R, Takala J, et al. The SOFA (Sepsis-related Organ Failure Assessment) score to describe organ dysfunction/failure. On behalf of the Working Group on Sepsis-Related Problems of the European Society of Intensive Care Medicine. Intensive Care Med. 1996;22:707-710.

12. Horan TC, Andrus M, Dudeck MA. CDC/NHSN surveillance definition of health care-associated infection and criteria for specific types of infections in the acute care setting. Am J Infect Control. 2008;36: 309-332.

13. Deschepper R, Grigoryan L, Lundborg CS, et al. Are cultural dimensions relevant for explaining cross-national differences in antibiotic use in Europe? BMC Health Serv Res. 2008;8:123.

14. Kaki R, Elligsen M, Walker S, Simor A, Palmay L, Daneman N. Impact of antimicrobial stewardship in critical care: a systematic review. J Antimicrob Chemother. 2011;66:1223-1230.

15. Bloos F, Hinder F, Becker K, et al. A multicenter trial to compare blood culture with polymerase chain reaction in severe human sepsis. Intensive Care Med. 2010;36:241-247.

16. Christ-Crain M, Stolz D, Bingisser R, et al. Procalcitonin guidance of antibiotic therapy in community-acquired pneumonia: a randomized trial. Am J Respir Crit Care Med. 2006;174:84-93.

17. Schuetz P, Christ-Crain M, Thomann R, et al. Effect of procalcitoninbased guidelines vs standard guidelines on antibiotic use in lower respiratory tract infections: the ProHOSP randomized controlled trial. JAMA. 2009;302:1059-1066.

18. Stolz D, Christ-Crain M, Bingisser R, et al. Antibiotic treatment of exacerbations of COPD: a randomized, controlled trial comparing procalcitonin-guidance with standard therapy. Chest. 2007;131: 9-19.

19. Bouadma L, Luyt CE, Tubach F, et al. Use of procalcitonin to reduce patients' exposure to antibiotics in intensive care units (PRORATA trial): a multicentre randomised controlled trial. Lancet. 2010;375: 463-474.

20. Nobre V, Harbarth S, Graf JD, Rohner P, Pugin J. Use of procalcitonin to shorten antibiotic treatment duration in septic patients: a randomized trial. Am J Respir Crit Care Med. 2008;177: 498-505.

21. Stolz D, Smyrnios N, Eggimann P, et al. Procalcitonin for reduced antibiotic exposure in ventilator-associated pneumonia: a randomised study. Eur Respir J. 2009;34:1364-1375.

22. Franzetti F, Antonelli M, Bassetti M, et al. Consensus document on controversial issues for the treatment of hospital-associated pneumonia. Int J Infect Dis. 2010;14(suppl 4):S55-S65. 
23. Niederman MS, Craven DE, Bonten MJ et al. Guidelines for the management of adults with hospital-acquired, ventilator-associated, and healthcare-associated pneumonia. Am Respir Crit Care Med. 2005; 171:388-416.

24. Montravers P, Dupont H, Gauzit R, et al. Strategies of initiation and streamlining of antibiotic therapy in 41 French intensive care units. Crit Care. 2011;15:R17.

25. Castle M, Wilfert CM, Cate TR, Osterhout S. Antibiotic use at Duke University Medical Center. JAMA. 1977;237:2819-2822.

26. Maki DG, Schuna AA. A study of antimicrobial misuse in a university hospital. Am J Med Sci. 1978;275:271-282.
27. Hecker MT, Aron DC, Patel NP, Lehmann MK, Donskey CJ. Unnecessary use of antimicrobials in hospitalized patients: current patterns of misuse with an emphasis on the antianaerobic spectrum of activity. Arch Intern Med. 2003;163:972-978.

28. Davey P, Brown E, Fenelon L, et al. Interventions to improve antibiotic prescribing practices for hospital inpatients. Cochrane Database Syst Rev. 2005;CD003543.

29. Vogelaers D, De Bels D, Foret F, et al. Patterns of antimicrobial therapy in severe nosocomial infections: empiric choices, proportion of appropriate therapy, and adaptation rates-a multicentre, observational survey in critically ill patients. Int J Antimicrob Agents. 2010;35:375-381. 